\title{
Stability of relativistic neutron stars in binary orbit
}

\author{
T. W. Baumgarte, ${ }^{1}$ G. B. Cook, ${ }^{2}$ M. A. Scheel, ${ }^{2}$ S. L. Shapiro, ${ }^{1,3}$ and S. A. Teukolsky ${ }^{2,4}$ \\ ${ }^{1}$ Department of Physics, University of Illinois at Urbana-Champaign, Urbana, Illinois 61801 \\ ${ }^{2}$ Center for Radiophysics and Space Research, Cornell University, Ithaca, New York 14853 \\ ${ }^{3}$ Department of Astronomy and NCSA, University of Illinois at Urbana-Champaign, Urbana, Illinois 61801 \\ ${ }^{4}$ Departments of Physics and Astronomy, Cornell University, Ithaca, New York 14853
}

(Received 12 May 1997; published 28 April 1998)

\begin{abstract}
We analyze the stability of relativistic, quasi-equilibrium binary neutron stars in synchronized circular orbit. We explore stability against radial collapse to black holes prior to merger, and against orbital plunge. We apply theorems based on turning points along uniformly rotating sequences of constant angular momentum and rest mass to locate the onset of secular instabilities. We find that inspiraling binary neutron stars are stable against radial collapse to black holes all the way down to the innermost stable circular orbit. [S0556-2821(98)02212-7]

PACS number(s): 04.40.Dg, 04.20.Ex, 04.25.Dm, 97.60.Jd
\end{abstract}

The two-body problem is one of the outstanding, unsolved problems in classical general relativity. However, neutron star binary systems are known to exist even within our own galaxy [1]. Binary systems are among the most promising sources for gravitational wave detectors now under construction, such as the Laser Interferometric Gravitational Wave Observatory (LIGO), VIRGO and GEO. This has motivated an intense theoretical effort to predict the gravitational waveform emitted during the inspiral and coalescence of two neutron stars.

Fully general relativistic treatments of the problem are complicated by the nonlinearity of Einstein's equations and the need for very large computational resources. Recently, Wilson and Mathews [2] reported preliminary results obtained with a relativistic numerical evolution code. Their dynamical calculations suggest that the neutron stars may collapse to black holes before their orbit becomes unstable and the stars plunge. Their results are in disagreement with predictions of Newtonian [3], post-Newtonian (PN) [4-6], perturbation [7], and analytic "local-asymptotic-rest-frame", $[8,9]$ calculations, all of which show that tidal fields stabilize neutron stars against radial collapse.

In a recent paper, we presented quasi-equilibrium, polytropic models of fully relativistic, equal mass neutron star binaries in synchronized circular orbits [10]. In Newtonian gravity, strict equilibrium exists for two stars in circular orbit. In general relativity, because of the emission of gravitational waves, binaries cannot be in strict equilibrium. However, outside of the innermost stable circular orbit (ISCO), the time scale for orbital decay by radiation is much longer than the orbital period, so that the binary can be considered to be in "quasi-equilibrium." This fact allows us to neglect both gravitational waves and wave-induced deviations from a circular orbit to a very good approximation. A detailed discussion of our approximations and numerical method will be presented in a forthcoming paper [11].

In [10], we focused on the construction of quasiequilibrium models, but did not discuss their stability. We found that the maximum allowed mass slightly increases as the separation of the stars decreases. Here, we construct sequences of constant angular momentum and sequences of constant rest mass, which then allows us to apply rigorous theorems based on turning-points along the sequences to locate the onset of secular instabilities. While we apply these theorems to our recent numerical results [10], the arguments presented in this paper are independent of our numerical method and can be applied to any sequence of quasiequilibrium neutron star binaries in synchronized orbit. We find that such binaries are stable against radial collapse to black holes until they encounter orbital instability at the ISCO.

Applying turning-point methods to binaries in corotation allows us to detect secular instabilities. The separation at which simultaneous extrema exist in the mass-energy and the angular momentum curves marks the point along an evolutionary sequence at which the binary becomes unstable in the presence of some dissipative mechanism. This instability is distinct from dynamical instability, which arises independently of any dissipation and grows on a dynamical time scale. Gravitational radiation can drive the secular instability [12], which is expected to occur before (i.e. at larger separation than) the onset of a dynamical instability $[3,13]$. It is therefore anticipated that it is the secular instabilities that limit the range of stable configurations.

Because of finite numerical resources, we cannot construct models of very large separation. We therefore observe the increase of the maximum mass only for separations smaller than about 4 stellar radii. However, even at this separation, tidal effects are still fairly small. Moreover, PN calculations, which are valid for larger separations, suggest that the maximum mass should also increase monotonically with decreasing separation $[4,6]$. We will therefore assume that the maximum allowed mass monotonically increases as the separation decreases.

For a given equation of state, cold equal mass binary neutron stars in synchronized circular orbit form a twoparameter family, just like single, uniformly rotating stars. In our numerical code we adopt the central density and relative separation to uniquely specify a particular configuration. A lemma by Friedman, Ipser and Sorkin [13], originally derived for single, uniformly rotating stars, can therefore be adapted to corotating binaries:

Lemma. Consider a two-parameter family of equal mass binary stars in synchronized circular orbits based on an equa- 
tion of state of the form $P=P\left(\rho_{0}\right)$. Suppose that along a continuous sequence of models labeled by a parameter $\lambda$ there is a point $\lambda_{0}$ at which both $\dot{M}_{0} \equiv d M_{0} / d \lambda$ and $\dot{J}$ vanish and where $d\left(\dot{\Omega} \dot{J}+\dot{\mu} \dot{M}_{0}\right) / d \lambda \neq 0$. Then the part of the sequence for which $\left(\Omega \dot{J}+\dot{\mu} \dot{M}_{0}\right)>0$ is unstable for $\lambda$ near $\lambda_{0}$.

Here $P$ is the pressure, $\rho_{0}$ the rest-mass density, $J$ and $M_{0}$ are the angular momentum and rest (baryon) mass of one of the two stars, $\Omega$ is the angular velocity and $\mu$ is the chemical potential. We will later use $M$ for the total mass-energy of one of the two stars [that is half the total Arnowitt-DeserMisner (ADM) mass of the spacetime]. The proof of the lemma follows directly from theorem 1 of [14]. In practice it is more useful to apply the following related theorem, which is an immediate consequence of the lemma [13]:

Theorem 1. Consider a continuous sequence of equal mass binary stars in synchronized circular orbit based on an equation of state of the form $P=P\left(\rho_{0}\right)$. Suppose that the total angular momentum is constant along the sequence, and that there is a point $\lambda_{0}$ where $\dot{M}_{0}=0$ [and where $\mu>0$, $\left.d\left(\dot{\mu} \dot{M}_{0}\right) / d \lambda \neq 0\right]$. Then the part of the sequence for which $\dot{\mu} \dot{M}_{0}>0$ is unstable for $\lambda$ near $\lambda_{0}$.

We can now apply this theorem to our numerical models. In these models the matter obeys a polytropic equation of state, $P=K \rho_{0}^{1+1 / n}$, where $K$ is constant and $n$ is the polytropic index. Note that physical dimensions enter the problem only through $K$. It is therefore convenient to adopt geometrized units with $G \equiv c \equiv 1$ and introduce the dimensionless quantities $\quad \bar{\rho}_{0}=K^{n} \rho_{0}, \quad \bar{M}_{0}=K^{-n / 2} M_{0}$, $\bar{M}=K^{-n / 2} M$ and $\bar{J}=K^{-n} J$ (see [15]). Numerical values in cgs units can easily be obtained by inserting appropriate values for $n$ and $K$, and restoring $G$ and $c$.

In Fig. 1 we plot $\bar{M}_{0}$ as a function of the central density $\bar{\rho}_{\mathrm{c}}$ for $n=1$. The dotted lines are curves of constant relative separation $z_{A}=r_{\text {in }} / r_{\text {out }}$. Here $r_{\text {in }}$ is the inner (coordinate) distance between the two stars, as measured between the two closest points along the axis, and $r_{\text {out }}$ is the outer distance, as measured between the most distant points along the axis. For stars in contact $z_{A}=0$, and for infinitely separated stars $z_{A}=1$. The lowest dotted line in Fig. 1 is for stars at a separation $z_{A}=0.3$, and the highest curve is for stars in contact. We expect that the Oppenheimer-Volkoff curve for infinitely separated stars lies below all these lines [16], such that the equilibrium mass supported by a given central density monotonically increases with decreasing separation.

Evolutionary inspiral sequences conserve the rest mass $\bar{M}_{0}$ and therefore follow horizontal lines in Fig. 1. This shows, for example, that the central density $\bar{\rho}_{0}$ decreases as the two stars approach each other.

The solid lines in Fig. 1 are contours of constant total angular momentum $\bar{J}$. The angular momentum of infinitely separated binaries is infinite, and decreases for approaching binaries. There is a critical angular momentum $\bar{J}^{\text {crit }}$, for which the contours form a saddle point (point A). This critical angular momentum and the critical mass $\bar{M}_{0}^{\text {crit }}$ at the saddle point take values which, like the maximum mass of nonrotating isolated stars, depend only on the polytropic index. For $n=1$ we find $\bar{J}^{\text {crit }} \simeq 0.05$ and $\bar{M}_{0}^{\text {crit }} \simeq 0.179$ [16]. The

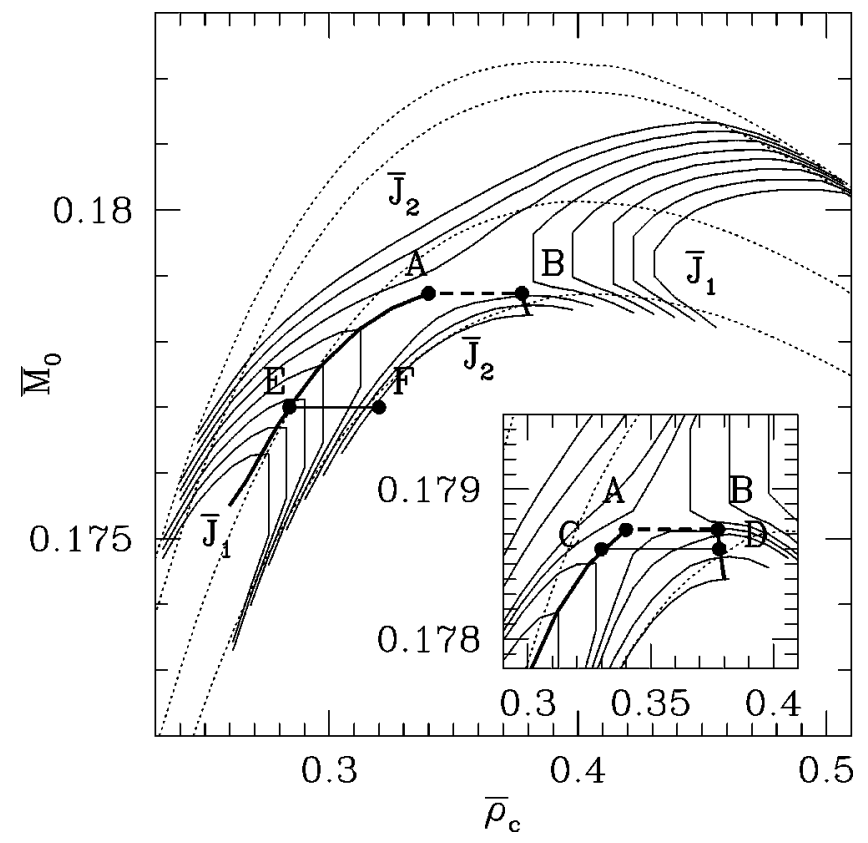

FIG. 1. Contours of constant angular momentum in a rest-mass versus central density diagram. The dotted lines are sequences of constant relative separation $z_{A}$, ranging from 0.3 (bottom curve) to 0 (touching stars, top curve). The thin solid lines are contours of constant angular momentum, ranging from $\bar{J}_{1}=0.049$ to $\bar{J}_{2}$ $=0.0498$ in increments of 0.0001 . In the inset we also show contours for $\bar{J}=0.04955$ and 0.049575 . The thick solid lines mark the onset of secular instabilities (see text).

saddle point divides the turning points of the contours into two separate classes: one for contours $\bar{J}<\bar{J}^{\text {crit }}$ and one for $\bar{J}>\bar{J}^{\text {crit }}$. We will identify the former with the instability at the ISCO, and the latter with a radial instability of supramassive stars at large separations.

The class for $\bar{J}<\bar{J}^{\text {crit }}$ exists for all rest masses $\bar{M}_{0}$ $<\bar{M}_{0}^{\text {crit }}$ and ends at the saddle point A. The physical interpretation of these turning points can be understood by following an evolutionary sequence for a coalescing binary of fixed rest mass, which starts at a large separation, e.g., at point $\mathrm{F}$. It evolves along a horizontal line, and initially both the angular momentum and the central density decrease with the separation. At point $\mathrm{E}$, however, the angular momentum goes through a minimum. According to the relation [18]

$$
d M=\Omega d J+\mu d M_{0},
$$

this minimum has to coincide with an extremum in the total mass-energy $\bar{M}$. This extremum is well understood in Newtonian gravity (e.g. [3]), PN theory (e.g. [19]), and general relativity [10], and marks the onset of an orbital instability at the ISCO. Note that this orbital instability is the first instability that these binaries encounter during inspiral.

For $\bar{J}>\bar{J}^{\text {crit }}$ we find a second class of turning points. This class starts at point B in Fig. 1 and ends at the maximum of the Oppenheimer-Volkoff (OV) curve for isolated, nonrotating stars $[16,17]$. The maximum on this limiting curve marks the onset of radial instability for isolated, nonrotating stars, and defines their maximum allowed rest mass $\bar{M}_{0}^{\max }$. By 


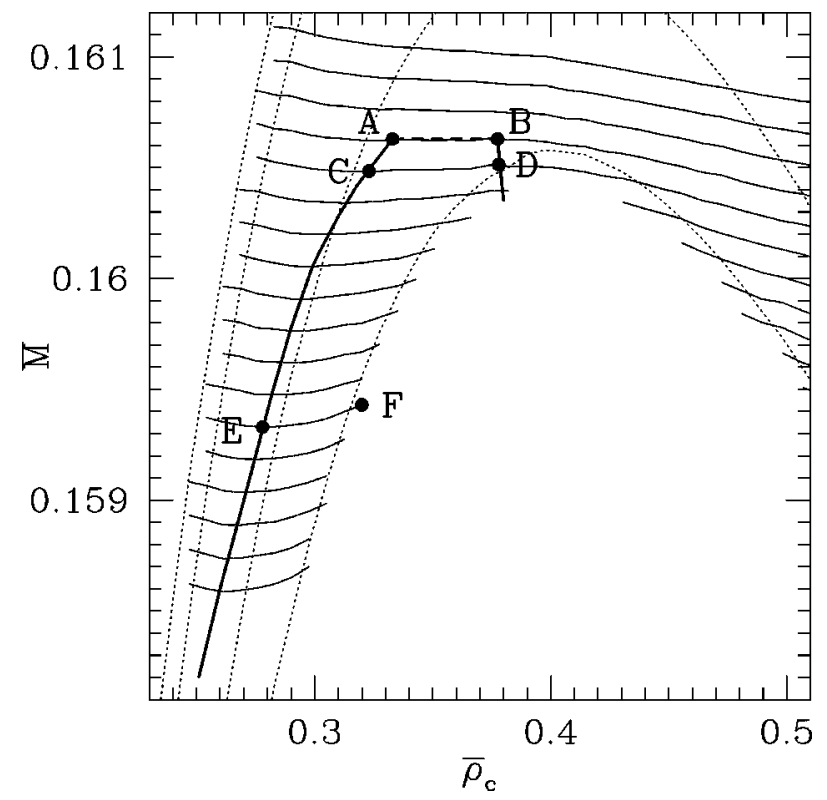

FIG. 2. Contours of constant rest mass in a mass-energy versus central density diagram. The dotted lines are sequences of constant relative separation $z_{A}$, ranging from 0.3 (bottom curve) to 0 (stars in contact, top curve). The thin solid lines are contours of constant rest mass, ranging from $\bar{M}_{0}=0.176$ to 0.1794 in increments of 0.0002. The thick solid lines mark the onset of secular instabilities (see text)

continuity, this class thus marks the onset of radial instability for supramassive sequences, consisting of stars with restmasses $\bar{M}_{0}^{\max }<\bar{M}_{0}<\bar{M}_{0}^{\text {crit }}$. Consider, for example, a binary somewhere on the line between points $\mathrm{C}$ and D. Following the inspiral of these stars, they will eventually encounter the ISCO at point C. If we could reverse the inspiral and follow an evolutionary sequence towards larger separations, the stars eventually have to become unstable and collapse to black holes, since their masses cannot be supported when in isolation. This will happen at turning point $\mathrm{D}$.

Note that so far we have only located turning points of contours of constant angular momentum. In order to meet all the conditions of theorem 1 and rigorously establish the onset of a secular instability, we also need to examine the chemical potential $\mu$. The chemical potential is not normally evaluated by a code that constructs equilibrium models (although it could be). Accordingly, it is useful to apply a different version of the theorem that does not refer to $\mu$ :

Theorem 2. Consider a continuous sequence of equal mass binary stars in synchronized circular orbit based on an equation of state of the form $P=P\left(\rho_{0}\right)$. Suppose that the rest mass $M_{0}$ is constant along the sequence, and that there is a point $\lambda_{0}$ where $\dot{M}=0$ [and where $\Omega>0, d(\Omega \dot{M}) / d \lambda \neq 0$ ]. Then the part of the sequence for which $\Omega \dot{M}>0$ is unstable for $\lambda$ near $\lambda_{0}$.

The proof follows from the lemma together with relation (1) (see also [20]). Note that according to Eq. (1) the extrema in theorems 1 and 2 have to coincide.

In Fig. 2 we plot contours of constant rest mass $\bar{M}_{0}$ in an $\bar{M}$ versus $\bar{\rho}_{\mathrm{c}}$ diagram. In this plot the contours describe secular evolutionary sequences. The contours again have two qualitatively different classes of extrema. One class is com-
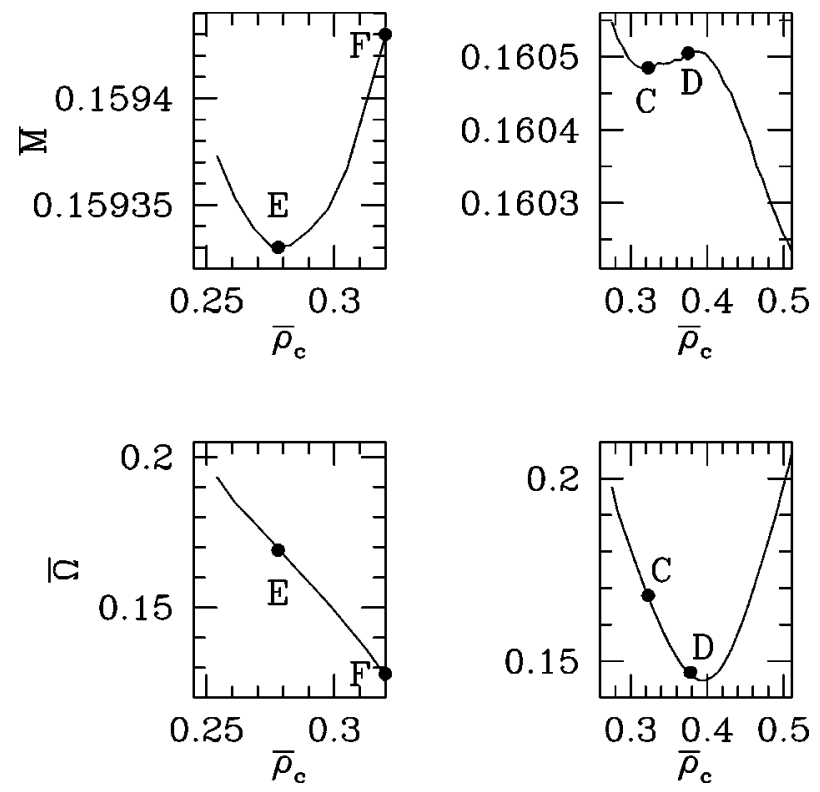

FIG. 3. Contours of constant rest mass. The top panels show two contours from Fig. 2, and the bottom panels show the corresponding angular velocities.

posed of minima of the total energy (e.g., point C), and the other is composed of maxima (point D). The former can again be associated with the onset of an orbital instability at the ISCO, while the latter marks the onset of radial instability of supramassive sequences at large separations. Both extrema end at the critical contour $\bar{M}_{0}^{\text {crit }}$, above which the contours are monotonically decreasing for increasing $\rho_{\mathrm{c}}$. The value of $\bar{M}_{0}^{\text {crit }}$ agrees with the height of the saddle point A and point B in Fig. 1. All extrema in Figs. 1 and 2 agree to within a few percent, which is a measure of the accuracy of our numerical code.

In order to verify the assumptions in theorem 2 , we have to make sure that the extrema of the constant rest mass contours do not coincide with an extremum of the angular velocity. In Fig. 3 we show the two contours connecting points E,F, and C,D, together with corresponding plots of $\bar{\Omega}$.

The minima at $\mathrm{E}$ and $\mathrm{C}$ can by analyzed very easily. Using the central density as the parameter $\lambda$, all the assumptions, $\dot{M}=0, \Omega>0$ and $d(\dot{\Omega} \dot{M}) / d \lambda \neq 0$, are satisfied, and we conclude that the configurations to the left of the minima, for which $\dot{\Omega} \dot{M}>0$, are unstable. These minima mark the onset of a secular instability close to the ISCO.

The maximum at $\mathrm{D}$ is a little less obvious from our numerical data, since it occurs very close to a minimum in $\bar{\Omega}$. However, the offset is measurable and larger than our numerical error, and we consistently find this offset. In addition, we know that supramassive stars must become unstable when moved out from finite separation to infinite separation. We therefore conclude that the extrema in $\bar{M}$ and $\bar{\Omega}$ are indeed distinct, so that we can apply theorem 2 . This establishes the onset of a secular radial instability for the maxima on the thick line extending downward from point B in Figs. 1 and 2.

We conclude that all binary configurations in the area under the thick solid lines and dashes in Figs. 1 and 2 are stable. This area can be subdivided into a normal and a su- 
pramassive region. All sequences with rest masses below the maximum allowed rest mass $\bar{M}_{0}^{\max }$ of an isolated, nonrotating star are normal sequences. These sequences start at finite separation (e.g., F) and end at the ISCO (e.g. E). In addition to these normal sequences, there are supramassive sequences with rest masses between $\bar{M}_{0}^{\max }$ and $\bar{M}_{0}^{\text {crit }}$. Evolutionary curves for these sequences connect a radial instability at large separation (e.g. D) with an orbital instability at the ISCO (e.g. C). We conclude that all normal binary neutron stars in synchronized orbit, with rest masses below the maximum for isolated, nonrotating configurations, are secularly stable against radial collapse to black holes down to the ISCO. At the ISCO they encounter a secular orbital instability.

Supramassive stars are unstable to collapse at large separation, but if formed at finite separation, remain stable as they inspiral down to the ISCO. Supramassive stars could arise in principle if a rapidly rotating, supermassive core were to undergo bifurcation at a finite radius. Alternatively, they could arise if two spinning neutron stars become bound in a binary or if two neutron stars accrete matter while in a binary.

The secular instability evolves on the same time scale as the inspiral, since both are driven by gravitational radiation $[3,12]$. Orbital plunge will occur at the onset of dynamical instability, which defines the true ISCO. Moreover, realistic binaries are more likely to be closer to irrotational (circulation $=0$ ) than corotational [21]. For such systems, however, the location of the dynamical instability is quite close to the onset of the secular instability, both in Newtonian theory [3] and in PN theory [6]. The onset of the dynamical instability is at slightly smaller separation.

The fact that dynamical instabilities typically arise at smaller separation than secular instabilities, together with the absence of a secular instability against radial collapse, up to the ISCO, suggests that a dynamical instability against radial collapse may also be absent in normal binary neutron stars.

This work was supported by NSF Grant AST 96-18524 and NASA Grant NAG 5-3420 at Illinois, and NSF Grant PHY 94-08378 at Cornell.
[1] J. H. Taylor and J. M. Weisberg, Astrophys. J. 345, 434 (1989); S. E. Thorsett, Z. Arzoumanian, M. M. McKinnon and J. H. Taylor, ibid. 405, L29 (1993).

[2] J. R. Wilson and G. J. Mathews, Phys. Rev. Lett. 75, 4161 (1995): J. R. Wilson, G. J. Mathews and P. Marronetti, Phys. Rev. D 54, 1317 (1996).

[3] D. Lai, F. A. Rasio, and S. L. Shapiro, Astrophys. J., Suppl. Ser. 88, 205 (1993).

[4] D. Lai, Phys. Rev. Lett. 76, 4878 (1996).

[5] A. D. Wiseman, Phys. Rev. Lett. 79, 1189 (1997).

[6] J. C. Lombardi, F. A. Rasio and S. L. Shapiro, Phys. Rev. D 56, 3416 (1997).

[7] P. R. Brady and S. A. Hughes, Phys. Rev. Lett. 79, 1186 (1997)

[8] É. É. Flanagan, gr-qc/9706045, 1997.

[9] K. S. Thorne, Phys. Rev. D (to be published), gr-qc/9706057.

[10] T. W. Baumgarte, G. B. Cook, M. A. Scheel, S. L. Shapiro and S. A. Teukolsky, Phys. Rev. Lett. 79, 1182 (1997).

[11] T. W. Baumgarte, G. B. Cook, M. A. Scheel, S. L. Shapiro and S. A. Teukolsky, Phys. Rev. D (to be published).

[12] S. Chandrasekhar, Astrophys. J. 161, 561 (1970).

[13] J. L. Friedman, J. R. Ipser and R. D. Sorkin,
Astrophys. J. 325, 722 (1988).

[14] R. D. Sorkin, Astrophys. J. 257, 847 (1982).

[15] G. B. Cook, S. L. Shapiro, and S. A. Teukolsky, Astrophys. J. 422, 227 (1994).

[16] Computing the Oppenheimer-Volkoff curve with a very high radial resolution yields a maximum mass which is slightly larger than the masses of some of our binary models (see Fig. 2 in [10]). However, we know from convergence tests that we underestimate the masses of the binary configurations by a few percent as a result of our coarse numerical grid.

[17] It is difficult to precisely locate the extrema near the saddle point with our numerical data. It may very well be that $\mathrm{A}$ and B actually coincide. However, this would not affect any of our conclusions about stability.

[18] J. B. Hartle, Astrophys. J. 161, 111 (1970); J. M. Bardeen, in Black Holes, edited by C. DeWitt (Gordon and Breach, New York, 1972).

[19] M. Shibata, Prog. Theor. Phys. 96, 317 (1996).

[20] G. B. Cook, S. L. Shapiro and S. A. Teukolsky, Astrophys. J. 398, 203 (1992).

[21] L. Bildsten and C. Cutler, Astrophys. J. 400, 175 (1992); C. S. Kochanek, ibid. 398, 234 (1992). 